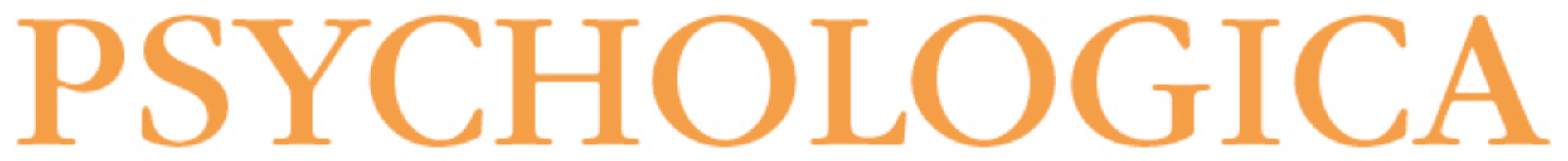

Vinculação, memórias de infância e estilos defensivos na população dependente de substâncias: estudo comparativo e multivariado

Autor(es): $\quad$ Carriço, Cláudia Nunes; Paixão, Rui

Publicado por: Imprensa da Universidade de Coimbra

URL

persistente: URI:http://hdl.handle.net/10316.2/3485

DOI: ～DOl:http://dx.doi.org/10.14195/1647-8606_52-2_24

Accessed : $\quad$ 26-Apr-2023 13:09:06

A navegação consulta e descarregamento dos títulos inseridos nas Bibliotecas Digitais UC Digitalis, UC Pombalina e UC Impactum, pressupõem a aceitação plena e sem reservas dos Termos e Condições de Uso destas Bibliotecas Digitais, disponíveis em https://digitalis.uc.pt/pt-pt/termos.

Conforme exposto nos referidos Termos e Condições de Uso, o descarregamento de títulos de acesso restrito requer uma licença válida de autorização devendo o utilizador aceder ao(s) documento(s) a partir de um endereço de IP da instituição detentora da supramencionada licença.

Ao utilizador é apenas permitido o descarregamento para uso pessoal, pelo que o emprego do(s) título(s) descarregado(s) para outro fim, designadamente comercial, carece de autorização do respetivo autor ou editor da obra.

Na medida em que todas as obras da UC Digitalis se encontram protegidas pelo Código do Direito de Autor e Direitos Conexos e demais legislação aplicável, toda a cópia, parcial ou total, deste documento, nos casos em que é legalmente admitida, deverá conter ou fazer-se acompanhar por este aviso.

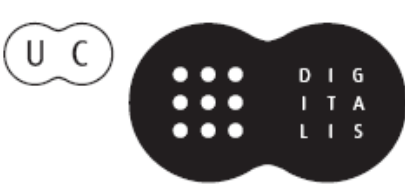




\section{NÚMERO 52}

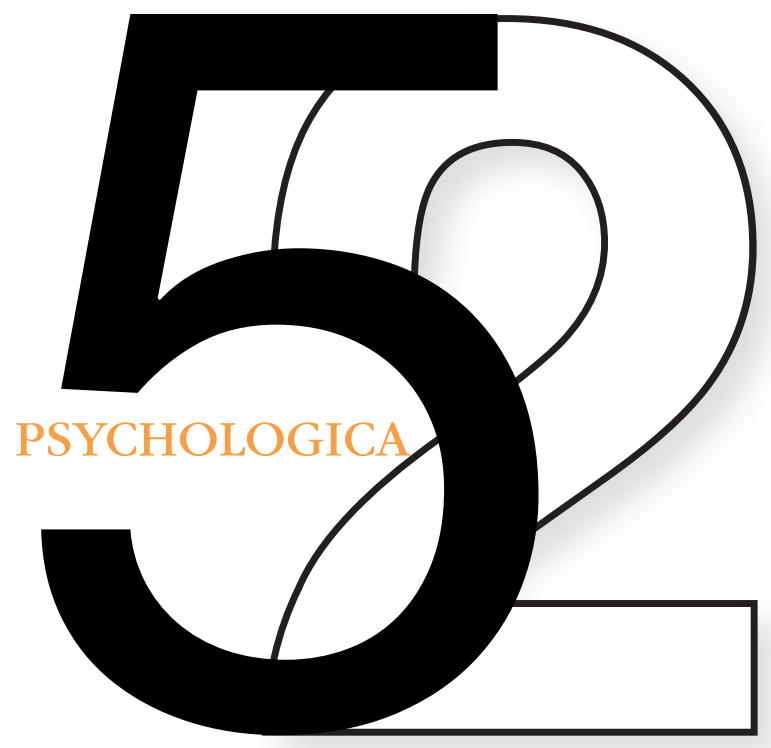

VOLUME II

IMPRENSA DA UNIVERSIDADE DE COIMBRA

FACULDADE DE PSICOLOGIA E DE CIÊNCIAS DA EDUCAÇÃo DA UNIVERSIDADE DE COIMBRA 


\title{
Vinculação, Memórias de Infância e Estilos Defensivos na População Dependente de Substâncias: Estudo Comparativo e Multivariado
}

\author{
Cláudia Nunes Carriço' \& Rui Paixão²
}

\begin{abstract}
Este artigo tem como objectivo avaliar as dimensões da vinculação na população dependente de substâncias (DS), bem como as variáveis associadas a este construto, particularmente as memórias de infância associadas à relação com os pais e os mecanismos de defesa. Numa primeira fase é feita a comparação entre um grupo de DS ( $n=65)$ e um grupo de sujeitos da população geral $(n=65)$ emparelhados ao nível do género, idade e escolaridade. As diferenças entre grupos mostraram-se significativas ao nível da vinculação, memórias de infância e mecanismos de defesa específicos, como a clivagem e a supressão. Numa segunda fase, estuda-se a relação entre estas variáveis no grupo DS, tendo os resultados mostrado que a memória da rejeição da mãe e a clivagem têm um efeito preditor ao nível das dimensões da vinculação estudadas, sendo a clivagem o estimador mais consistente destas dimensões.
\end{abstract}

PALAVRAS-CHAVE: Vinculação, Modelos Internos Dinâmicos, Dependência de Substâncias, Memórias de Cuidados Parentais, Estilos Defensivos

\section{Introdução}

A partir da relação com a figura de vinculação precoce, a criança começa a estruturar a sua vida psíquica, organizando representações mentais dinâmicas (modelo interno dinâmico de si e do outro), construídas com base nas experiências com o cuidador precoce. A sensitividade e a responsividade são aspectos do cuidador, determinantes para o desenvolvimento destes padrões: a criança irá usar as representações mentais que derivam das relações precoces como guias heurísticos para interacções futuras (Bowlby, 1958, 1980). Estes modelos internos têm componentes afectivas e cognitivas e permitem prever e interpretar o comportamento dos outros, estando na base do desenvolvimento da capacidade de mentalização tal como definida por Fonagy (2001).

\footnotetext{
1 Psicóloga Clínica - Îris_murdoch@live.com.pt

2 Faculdade de Psicologia e de Ciências da Educação da Universidade de Coimbra - rpaixao@fpce.uc.pt
} 
No contexto destes processos de vinculação, Bowlby (1980) associa a estratégia primária de regulação emocional, isto é, a aproximação da figura de vinculação, ao padrão de vinculação seguro, a que estão subjacentes modelos internos dinâmicos positivos de si, do outro e da relação. Se, contudo, o indivíduo não valorizar a presença do outro como capaz de responder às suas necessidades (modelo interno dinâmico do outro negativo), ou não se considerar digno de ser amado e protegido (modelo interno dinâmico de si negativo), irá utilizar estratégias secundárias de hiperactivação ou desactivação do sistema de vinculação, associadas respectivamente aos padrões ansioso e evitante. A hiperactivação refere-se a esforços intensos para manter a proximidade com a figura de vinculação e assegurar a sua atenção e protecção. A desactivação refere-se à inibição da procura de proximidade e supressão, ou desvalorização das ameaças que possam activar o sistema de vinculação (Shaver \& Milkulincer, 2005).

Os maus-tratos físicos ou psicológicos na infância têm sido considerados como tendo um impacto negativo na vinculação e parecem estar na base dos padrões de vinculação não seguros no adulto (Levy, Blatt, \& Shaver, 1998), bem como do desenvolvimento posterior de fenómenos psicopatológicos (Fonagy, 2001). Os padrões de vinculação parecem ser, portanto, relativamente estáveis, mas é possivel que sofram reformulações ao longo da vida, na sequência de relações reparadoras, ou de experiências traumáticas como perdas de pessoas significativas, razão pela qual são definidos como dinâmicos (Bretherton, 1985). Esta estabilidade relativa tem sido compreendida de diversos modos, considerando, por exemplo, a manutenção do mesmo tipo de relações ao longo da vida (Fonagy, 2001) ou, ainda, a actualização das representações mentais das relações, que se constroem a partir das experiências precoces internalizadas, no contexto dos relacionamentos posteriores (Bowlby, 1958, 1988; Fonagy, 2001). De qualquer modo, as relações precoces marcadas pelo medo e maus-tratos têm, desde os trabalhos de Main e Hesse (1990), sido relacionadas com o padrão de vinculação desorganizado, tal como é avaliado pelo Adult Attachment Interview (AAI) (Main, Kaplan, \& Cassidy, 1985). Nestes casos, a figura de vinculação parece ser simultaneamente sinal de segurança e de perigo, estando na base de experiências precoces associadas a desenvolvimentos psicopatológicos (e.g. Bernstein, Stein, \& Handelsman, 1998; Pauli-Pott \& Mertesacker, 2009; Rodrigues et al., 2004). É nesta base que as relações entre o padrão de vinculação e a perturbação dissociativa da identidade, a ansiedade, o stress pós-traumático, a somatização e o abuso de substâncias têm sido estudados (e.g. Caspers, Yucuis, Troutman, \& Spinks, 2006; Fonagy, Leigh, Steele, Kennedy, \& Matoon, 1996; Mikulincer \& Shaver, 2007; Riggs et al., 2007; Riggs \& Jacobvitz, 2002; Rosenstein \& Horowitz, 1996; Schindler, Thomasius, Petersen, \& Sack, 2009; Schindler et al., 2005). 


\section{Vinculação e Dependência de Substâncias}

A dependência de substâncias (DS) (DSM-IV-TR), no contexto da vinculação, tem sido amplamente estudada (e.g. Fonagy et al., 1996; McNally, Palfai, Levine, \& Moore, 2003; Schindler et al., 2009; Schindler et al., 2005), relacionando-a, retrospectivamente, com vivências negligentes das necessidades de vinculação, compensadas artificialmente com a procura de sensações de fusão e tranquilidade obtidas com o consumo de drogas (Schindler et al., 2009). Estes estudos mostram uma menor prevalência de padrões não seguros de vinculação nestas populações, embora os resultados sejam relativamente inconsistentes. Isto, no entanto, pode estar relacionado com a utilização de diferentes tipos de medidas, nomeadamente a já referida AAI, que avalia representações mentais relacionadas com a vinculação e as medidas de auto-resposta, que avaliam "estilos de vinculação", como o Attachment Style Questionaire (ASO) (Hazan \& Shaver, 1987) e o Modelo de Quatro Categorias de Bartholomew e Horowitz (1991). Estes dois grandes tipos de medidas representam duas linhas de investigação diferentes, com resultados nem sempre concordantes. Por exemplo, os resultados obtidos com a AAI associam a DS ao padrão evitante e ansioso (Rosenstein \& Horowitz, 1996), ao padrão desorganizado (Rigss \& Jacobvitz, 2002) e ao padrão ansioso e desorganizado (Fonagy et al., 1996). Os estudos com o ASO mostram uma prevalência do padrão evitante (Finzi-Dottan, Cohen, Iwaniec, Sapir, \& Wietzman, 2003; Mickelson, Kessler, \& Shaver, 1997), embora outros estudos evidenciem que o padrão ansioso se associa fortemente ao consumo de álcool em adolescentes (Cooper, Shaver, \& Collins, 1998). Thorberg e Lyvers (2006), utilizando o Adult Attachment Scale (AAS) ${ }^{3}$ (Collins, 1996), verificaram que os sujeitos com DS obtinham pontuações mais altas na dimensão ansiedade e mais baixas na dimensão conforto com a proximidade. Os estudos que utilizaram o Modelo de Quatro Categorias mostraram uma associação entre um modelo interno negativo do self e o consumo de álcool (McNally et al., 2003) e uma prevalência do padrão amedrontado entre sujeitos com DS (Schindler et al., 2005).

Teoricamente, as estratégias de desactivação do sistema de vinculação (desinvestimento nas relações e desvalorização das ameaças) associadas ao padrão evitante têm um objectivo semelhante ao do consumo de drogas, se se pensar em termos de auto-medicação e anestesia. No entanto, há também que ter em conta que quanto mais bem sucedidas são as estratégias de desactivação, menor será a necessidade de consumir uma substância psicoactiva para a regulação do afecto. Numa perspectiva divergente, Cooper et al. (1998) propõem que os adolescentes ansiosos/ambivalentes são os mais vulneráveis a comportamentos de "passagem ao acto", onde se inclui a DS, por serem mais sensíveis à pressão dos 
pares, contrastando com adolescentes evitantes que não se envolvem com o grupo e, portanto, se mostram mais relutantes em experimentar substâncias. Contudo, uma vez iniciado o comportamento de consumo, os adolescentes classificados como evitantes seriam mais vulneráveis que os seguros.

562 A inconsistência destes resultados pode estar ligada ao facto do consumo de drogas poder mascarar padrões de vinculação inicialmente ansiosos, activando e mimetizando estratégias associadas ao padrão evitante. Além disso, os comportamentos e modos de relacionamento associados ao consumo de substâncias podem ser responsáveis pela utilização de determinadas estratégias de vinculação, sendo que o padrão de vinculação na DS emerge dessas relações disfuncionais e não tanto dos modelos internos dinâmicos gerados na relação precoce. Ainda neste sentido tem sido sugerido que os conceitos medidos pelos questionários de auto-resposta são apenas relatos de experiências de vinculação e não os padrões de vinculação em si, característicos dos sujeitos (Bernier \& Dozier, 2002, cit. in Canavarro, Dias \& Lima, 2006). A utilização destes questionários pode, nalguns casos, levar à subestimação do padrão evitante, já que estes sujeitos tendem a reportar menos comportamentos problemáticos que os sujeitos ansiosos, do mesmo modo que tendem a apresentar menores taxas de adesão a programas de tratamento (Caspers et al., 2006). Assim, ainda que o padrão evitante apareça frequentemente associado à DS, esta associação poderá ser, na realidade, mais forte.

Outra razão possivel para a inconsistência dos resultados é relativa ao facto dos trabalhos referidos serem bastante heterogéneos em relação às amostras, incluindo a diversidade de problemáticas psicopatológicas (duplos diagnósticos) associadas a esses sujeitos e o tipo de substâncias consumidas (e.g. Cooper et al., 1998; Finzi-Dotan et al., 2003; Fonagy et al., 1996; Mickelson et al., 1997; Rosenstein \& Horowitz, 1996; Schindler et al., 2005).

De qualquer modo, a ligação conceptual mais bem estabelecida parece ser a que se verifica com o padrão de vinculação amedrontado, do modelo de quatro categorias de Bartholomew e Horowitz (1991). Nestes casos, as defesas evitantes (de desactivação) parecem não ser eficazes, particularmente em situações de sofrimento intenso. O consumo da substância seria compreendido como um substituto artificial para estas defesas (desvalorização de ameaças e inibição de procura de proximidade), na impossibilidade de regular o afecto através da aproximação de uma figura securizante (Schindlder et al., 2005).

Outros aspectos em estudo neste domínio envolvem as experiências precoces, particularmente os maus-tratos, e as representações mentais que estes indivíduos têm dos seus cuidadores (Branstetter, Bower, Kamien, \& Amass, 2008). As medidas mais utilizadas em estudos deste tipo são o Inventory for Assessing Memories of Parental Rearing Behavior (EMBU) (Perris, Jacobson, Lindstorm, von Knorring, \& Perris, 1980) e 
o Parental Bonding Instrument (PBI) (Parker, Tupling, \& Brown, 1979). Os resultados mostram que os sujeitos com DS recordam os cuidados parentais como marcados pela rejeição, falta de suporte emocional e controlo rígido (Emmelkamp \& Heeres, 1988; Shweitzer \& Lawton, 1989), com diferenças mais evidentes para a rejeição e falta de suporte emocional por parte do pai (De Jong, Harvefeld, van der Wlelen, $\&$ van der Stank, 1991), estilo parental rígido e severo (Zimmer-Hoffer \& Kooyman, 1996), ou simultaneamente rejeitante e de sobreprotecção (Andersson \& Eiseman, 2003). Nos estudos com o EMBU, curiosamente, são as dimensões relativas ao pai que parecem ter maior peso e apresentar diferenças mais marcadas. De um modo mais geral, os sujeitos DS sentem os pais como frios, indiferentes, controladores e intrusivos, num estilo de cuidados precoces que tem sido descrito na literatura como affectionless control (controlo sem afecto) (e.g. Andersson \& Eiseman, 2003; De Jong et al., 1991; Emmelkamp \& Heeres, 1988; Shwitzer \& Lawton, 1989).

Um outro problema é relativo à comorbilidade do abuso de substâncias com outras perturbações. Neste caso, as associações mais frequentes têm sido descritas com a Perturbação Borderline da Personalidade, a Perturbação Bipolar, a Esquizofrenia e as Perturbações do Humor (Conrod \& Stewart, 2005). Quando associadas aos padrões de vinculação estas comorbilidades parecem implicar sentidos diferentes (Mickelson et al., 1997). Por outras palavras, nem sempre é claro se as diferenças no padrão de vinculação resultam do fenómeno psicopatológico, de interacções precoces traumáticas, do consumo de substâncias em si ou da interacção entre estas variáveis. Este problema é ainda mais importante quando se verifica que a DS se associa significativamente, de forma primária ou secundária, a outras perturbações psiquiátricas (Conrod \& Stewart, 2005; Teixeira \& Fernandes, 2005).

\section{Dependência de Substâncias e Mecanismos de Defesa}

Segundo a teoria da vinculação, a utilização de defesas psíquicas, tal como são concebidas pelas teorias psicodinâmicas actuais, é associada aos objectivos da utilização de estratégias de vinculação secundárias, isto é, a defesas que actuam quando a situação de conflito, sofrimento ou ameaça, não é resolvida com a aproximação à figura de vinculação (estratégia primária para lidar com essas dificuldades emocionais) (Shaver \& Milkulincer, 2005). No conjunto dessas defesas, Bowlby (1980) considerou a exclusão defensiva como a defesa central relacionada com os processos de vinculação, distinguindo-a de outros processos envolvidos na exclusão de material para processamento, em função do seu objectivo: o material é excluído para impedir que o indivíduo tenha consciência dos acontecimentos e pensamentos que seriam intoleráveis se fossem aceites como verdadeiros. Para além desta defesa, propõe um segundo processo defensivo, a que chama segregação de sistemas principais. O termo "sistema principal" denota uma capacidade do 
self para se auto-percepcionar e dirigir. Os selves segregados têm cada um a sua própria secção de armazenamento na memória, podendo alternar na consciência, embora um deles tenda a ser dominante em determinado tempo, enquanto o outro se encontra num estado de desactivação completa ou parcial. O sistema principal que se encontra desactivado é, ainda segundo Bowlby (1980), organizado e não menos consistente do que o sistema que está acessível à consciência e à acção. Este conceito de segregação de sistemas principais é, do ponto de vista teórico, semelhante ao conceito de clivagem (splitting) proposto por Kernberg (1977) e que o autor considera ser o principal mecanismo do funcionamento mental associado à patologia Borderline. De qualquer modo, os modelos internos dinâmicos contraditórios são codificados na memória de forma distinta, o que explicaria certos fenómenos observados na clínica, como as imagens uniformemente adversas dos pais contrapostas às memórias detalhadas mais favoráveis que certos pacientes revelam ao descrever esses mesmos pais (Bowlby, 1980).

Os estudos que procuram explorar ou validar as associações entre os estilos de vinculação e as defesas têm-se centrado, fundamentalmente, no conceito de "perfis defensivos", proposto pela American Psychiatric Association (2002), tentando associar os padrões de vinculação inseguros a clusters imaturos de estilos defensivos (Barone, Lingiardi, \& Maffei, 1996; Shaver \& Milkulincer, 2005).

No contexto da DS, a investigação com mulheres dependentes de opiáceos e alcoólicas verificou não existirem diferenças significativas entre os grupos em termos de defesas maturas; as mulheres alcoólicas, neste caso, utilizavam preferencialmente defesas neuróticas, enquanto as dependentes de opiáceos utilizavam, sobretudo, defesas imaturas, destacando-se a clivagem, negação, fantasia, isolamento e desvalorização (Raketic, Kovacevic, \& Djuric, 2009). Com o Defense Style Questionnaire (DSO) (Bond, Gardner, Christian, \& Sigal, 1983), Comings, McMurray, Johnson, Dietz e Muhleman (1995), verificaram que os sujeitos com DS diferem significativamente do grupo de controlo ao nível das defesas maturas, obtendo resultados significativamente mais baixos (particularmente no humor). Contudo, estes mesmos sujeitos DS não diferem significativamente dos sujeitos com Síndroma de Tourette, o que sugere que o nível defensivo pode ser uma medida global de ajustamento psicológico.

\section{Objectivos}

O presente estudo visa avaliar as relações entre as dimensões da vinculação, tal como são operacionalizadas pela escala de vinculação do adulto (EVA)4, as

4 Versão portuguesa da Adult Attachmente Scale-R (Canavarro et al., 2006; Collins \& Read, 1990). 
variáveis memórias de infância (EMBU)5 e os mecanismos de defesa (DSO-4O)6 em sujeitos DS e de controlo.

No modelo em estudo, parte-se da hipótese que as diferenças individuais na organização dos padrões de vinculação são uma função dos contextos precoces de interacção que organizam os modelos internos dinâmicos de si, do outro e da relação. Os padrões de vinculação não seguros associam-se a experiências de rejeição e inconsistência, que estão na origem de modelos internos dinâmicos negativos ou contraditórios (não integrados). Nestes casos, a estratégia primária de regulação do afecto, isto é, a aproximação da figura de vinculação, não é eficaz. Assim, os indivíduos não seguros utilizam estratégias de hiper e hipoactivação do sistema de vinculação, organizadas para reduzir a ansiedade, e que podem ser adaptativas no contexto inicial, mas desadaptativas no decurso do desenvolvimento (Canavarro et al., 2006). Também a incapacidade em regular as emoções negativas através do contacto com a figura de vinculação implica um nível de fragilidade que se revê na utilização de estilos defensivos mais imaturos e numa menor capacidade de mentalização (Fonagy, 2001). A fixação destes estilos defensivos mais imaturos pode ser um aspecto que permite compreender a eventual continuidade entre as experiências precoces, os padrões de vinculação na infância e a vinculação no adulto. No centro deste núcleo primitivo encontramos, por hipótese, a incapacidade em integrar os aspectos bons e maus do cuidador bem como do self, que assim permanecem segregados, tal como é evidenciado pelo uso da clivagem (splitting) . Na Figura 1 apresentam-se as relações conceptuais entre as referidas variáveis.

Os estudos entre estas variáveis incidiram, numa primeira fase, na medição do impacto da variável DS na variação das dimensões de vinculação, memórias de infância e mecanismos de defesa, através da comparação com uma amostra de controlo retirada da população geral. A sintomatologia psicopatológica foi controlada considerando-se o problema da comorbilidade.

Numa segunda fase, considerando apenas o grupo DS, a investigação teve como objectivo avaliar o tipo de relações entre as variáveis e esclarecer o possivel efeito preditor, no padrão de vinculação do adulto, das experiências precoces com as figuras de vinculação e dos mecanismos de defesa utilizados por estes sujeitos.

\footnotetext{
5 Versão portuguesa Canavarro (1996).

6 Versão portuguesa da Defense Style Questionaire-40 (Amaral, 2008; Bond et al., 1983).

7 Referimo-nos aqui à organização borderline ou estado-limite marcada por angústias depressivas de abandono, relações de objecto dependentes e mecanismos de defesa regressivos, particularmente a clivagem, a idealização primitiva e a negação (Braconnier et al., 2007; Kernberg, 1977).
} 


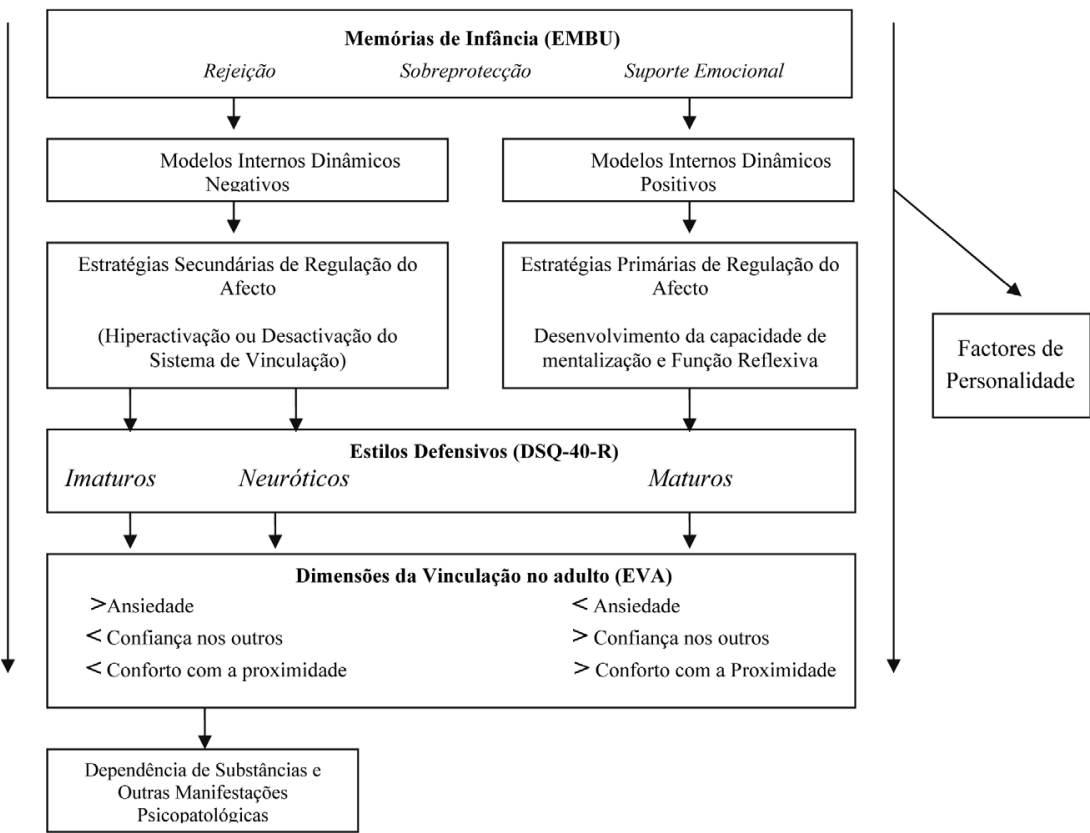

Figura 1 - Modelo das relações entre memórias de infância, estilos defensivos e dimensões da vinculação no adulto em sujeitos Dependentes de Substâncias

\section{Amostra e instrumentos}

A amostra em estudo é constituída por 130 sujeitos, divididos em dois grupos: grupo com dependência de substâncias (DS) $(n=65)$ e grupo de controlo (C) $(n=65)$. Os sujeitos do grupo DS constituem uma amostra de conveniência, tendo sido recrutados em instituições de tratamento, acolhimento ou de redução de riscos. Neste grupo, do total de sujeitos avaliados $(n=67)$, dois foram excluídos por apresentarem défices cognitivos acentuados, resultantes de AVC. No grupo $C$, foi critério de exclusão a existência de problemas conhecidos ou relatados de abuso/dependência de substâncias ao longo da vida e a existência de problemas com a justiça. Em ambos os grupos a prevalência de sintomatologia psicopatológica foi avaliada através da Versão Portuguesa do Brief Symtom Inventory (BSI) (Canavarro,1999; Derogatis \& Spencer, 1982).

A amostra do grupo $C$ foi emparelhada nas variáveis género $\left[\chi^{2}(n=130)=.000\right.$, $p=1.00]$, idade $\left[F_{(1,128)}=1.666, p=.199\right]$ e escolaridade $\left[F_{(1,128)}=2.230, p=.138\right]$. Os participantes que compõem este grupo foram recrutados em turmas de ensino nocturno ou profissional, tendo alguns sido pessoalmente contactados. A caracterização sócio-demográfica dos dois grupos é apresentada na Quadro 1. 
Os sujeitos de ambos os grupos responderam a um questionário sócio-demográfico para as variáveis género, idade, estado civil, anos de escolaridade completos, situação laboral actual e habitação. Do questionário fazia parte uma secção destinada a avaliar os consumos de substâncias ao longo da vida. De referir, ainda, que todos os sujeitos foram previamente informados dos objectivos da investigação, do carácter voluntário e confidencial da sua participação, da possibilidade de poderem desistir a qualquer momento e da necessidade de assinarem um termo de consentimento informado.

A maioria dos sujeitos do grupo DS, embora policonsumidores 8 , indica como droga principal a heroína $(60 \%, n=39)$, seguida da cocaína $(32.3 \%, n=21)$. Dois sujeitos apontam o haxixe (3.1\%), dois o LSD (3.1\%) e um o álcool (1.5\%). A droga inicial, para a maioria dos sujeitos da amostra, é o haxixe. A droga principal começou a ser consumida, em média, pelos 18.3 anos de idade. Têm em média 15.7 anos de consumo ( $D P=6.342$ ) e todos se encontram em tratamento: a grande maioria em Comunidade Terapêutica ( $n=47,72 \%)$, e a quase totalidade dos restantes $(n=17$, 26\%) numa Equipa de Tratamento com apoio psicológico regular. Apenas um dos sujeitos se encontra exclusivamente num programa com antagonista.

Quadro 1. Características sócio-demográficas do grupo DS $(n=65)$ e de Controlo $(n=65)$

\begin{tabular}{|c|c|c|c|c|c|}
\hline & & \multicolumn{2}{|c|}{$\begin{array}{c}\text { Grupo DS } \\
\text { (Toxicodependentes) }\end{array}$} & \multicolumn{2}{|c|}{$\begin{array}{c}\text { Grupo C } \\
\text { (Grupo de Controlo) }\end{array}$} \\
\hline & & $\mathrm{n}$ & $\%$ & $\mathrm{n}$ & $\%$ \\
\hline \multirow[t]{2}{*}{ Género } & Masculino & 53 & 81.5 & 53 & 81.5 \\
\hline & Feminino & 12 & 18.5 & 12 & 18.5 \\
\hline \multirow[t]{4}{*}{ Idade } & Mínimo & 20 & - & 20 & - \\
\hline & Máximo & 54 & - & 59 & - \\
\hline & Média & 34.154 & - & 32.269 & - \\
\hline & Desvio Padrão & 6.243 & - & 7.629 & - \\
\hline \multirow[t]{4}{*}{ Estado Civil } & Solteiro & 41 & 63.1 & 30 & 46.143 \\
\hline & Casado/união de facto & 14 & 21.6 & 27 & 41.438 \\
\hline & Viúvo & o & o & o & 0 \\
\hline & Divorciado/Separado & 10 & 15.408 & 8 & 12.308 \\
\hline \multirow{4}{*}{$\begin{array}{l}\text { Anos de } \\
\text { Escolaridade } \\
\text { Completos }\end{array}$} & Mínimo & 4 & - & 5 & - \\
\hline & Máximo & 18 & - & 17 & - \\
\hline & Média & 8.569 & - & 8.723 & - \\
\hline & Desvio-Padrão & 2.889 & - & 1.916 & - \\
\hline \multirow{5}{*}{$\begin{array}{l}\text { Situação } \\
\text { Laboral } \\
\text { Actual }\end{array}$} & Estudante & o & o & 4 & 6.154 \\
\hline & Trab. não qualificado & 5 & 7.7 & 23 & 35.385 \\
\hline & Desempregado & 60 & 92.3 & 23 & 35.385 \\
\hline & Serviços & o & 0 & 10 & 15.385 \\
\hline & Outro & o & o & 4 & 5.285 \\
\hline
\end{tabular}

8 Todos os sujeitos referem ser consumidores de, pelo menos, três das substâncias incluídas numa lista de 14 


\begin{tabular}{|c|c|c|c|c|c|}
\hline \multirow[t]{5}{*}{ Habitação } & Pais/familiares & 33 & 50.8 & 25 & 38.462 \\
\hline & Sozinho & 11 & 16.9 & 6 & 9.231 \\
\hline & $\begin{array}{l}\text { Cônjuge/Compan- } \\
\text { heiros }\end{array}$ & 17 & 26.1 & 24 & 36.923 \\
\hline & Filhos & o & 0.0 & 4 & 6.154 \\
\hline & Outros & 4 & 6.2 & 6 & 9.23 \\
\hline
\end{tabular}

Nota: Os sujeitos que se encontravam a residir em Comunidade Terapêutica (CT) responderam ao questionário considerando a sua situação antes de entrar na instituição (por exemplo, com quem viviam antes de entrar na CT e se estavam, ou não, nessa altura a trabalhar).

Em relação aos instrumentos é de salientar que, no caso da EVA, foram considerados os factores ansiedade, conforto com a proximidade e confiança nos outros. A partir das pontuações obtidas para cada um destes factores os sujeitos foram classificados nas categorias definidas por Bartholomew e Horowitz (1991). Neste caso, seguiu-se o método recomendado por Canavarro et al. (2006), tendo sido criado um novo factor denominado conforto-confiança, a partir da média da pontuação de cada sujeito nas dimensões confiança nos outros e conforto com a proximidade. No EMBU, versão abreviada (Arrindell, Emmelkamp, Monsma, \& Brilman, 1983), foram considerados os factores rejeição, suporte emocional e sobreprotecção. Com o DSO-40 foram avaliados os 18 correlatos conscientes dos mecanismos de defesa descritos no DSM-IV-TR mais os dois acrescentados pelos autores originais da escala: sublimação e antecipação. A versão portuguesa aqui aplicada (Amaral, 2008) inclui seis factores organizados dos "menos adaptativos" aos "mais adaptativos": F1 - defesas imaturas (projecção, agressão passiva, acting out, clivagem); F2 - defesas de distorção de imagem (desvalorização, isolamento, fantasia autística); F3 - defesas neuróticas 1 (supressão, negação, dissociação); F4 - defesas de encobrimento (racionalização, denegação); F5 - defesas neuróticas 2 (somatização, pseudo-altruísmo, formação reactiva, deslocamento, idealização); F6 - defesas maturas (sublimação, antecipação e humor).

\section{Apresentação dos resultados}

\subsection{Comparação entre grupos C e DS}

Em relação à sintomatologia psicopatológica, medida pelo $\mathrm{BSI}$, os resultados de ambos os grupos não se afastam de forma significativa dos valores encontrados

\footnotetext{
9 Os sujeitos foram classificados da seguinte forma: indivíduos com valores superiores ao valor 3 no factor conforto-confiança e valores inferiores a 3 no factor ansiedade foram classificados como seguros; os sujeitos com pontuações superiores a 3 nos dois factores (conforto-confiança e ansiedade) como preocupados; os sujeitos com pontuações inferiores a 3 nos dois factores foram classificados como evitantes e os que obtiveram valores superiores ao valor médio (3) no factor ansiedade e inferiores no factor conforto-confiança foram classificados como amedrontados.
} 
por Canavarro (1999), quer a nível dos Índices Globais, quer a nível das nove escalas que compõem esta medida. O grupo DS, inclusivamente, obtém resultados ligeiramente inferiores aos índices médios referidos por esta autora para a população com perturbação emocional.

Contudo, analisando as pontuações obtidas no BSI pelos dois grupos, conclui-se que os sujeitos do grupo DS obtêm pontuações significativamente mais altas em dois índices gerais: IGS $\left.\left[\mathrm{F}_{(1,128)}\right]=6.360, p=.013\right]$ e ISP $\left[\mathrm{F}_{(1,128)}=11.310, p=.001\right]$. A associação entre a variável grupo e a pontuação no IGS é pequena $\left(\eta^{2}=.047\right)$, podendo apenas $4.7 \%$ da variância nesta dimensão ser atribuída ao efeito grupo. No caso do ISP, $8.2 \%\left(\eta^{2}=.082\right)$ da variância pode ser atribuida ao efeito grupo, sendo a associação entre as variáveis moderada. O poder estatístico do estudo é grande no caso do ISP (.916), ultrapassando o critério standard (.80). No caso do IGS, o poder observado é inferior (.706). A nível das nove dimensões de psicopatologia, são encontradas diferenças entre os grupos nas subescalas obsessões-compulsões $\left[\mathrm{F}_{(1,128)}=5.379, p=.022\right]$, depressão $\left[\mathrm{F}_{(1,128)}=11.353, p<.001\right]$ e psicoticismo $\left[\mathrm{F}_{(1,128)}=\right.$ $28.305, p<.001]$, pontuando os sujeitos DS mais alto. A associação entre a variável grupo e a subescala psicoticismo é elevada $\left(\eta^{2}=.181\right)$, sendo $18.1 \%$ da variância encontrada nesta subescala explicada pela variável grupo. O poder observado neste caso atinge o valor máximo (1). A associação entre a depressão e a variável grupo é moderada $\left(\eta^{2}=.081\right)$ sendo $8.1 \%$ da variância nesta subescala atribuivel ao factor grupo. O poder estatístico atinge também um valor adequado. Quanto à subescala obsessões-compulsões, apenas $4 \%$ da variância observada se deve ao factor grupo, sendo a associação entre as variáveis pequena $\left(\eta^{2}=.040\right)$. O poder estatístico é baixo (.633).

Nos estudos prévios de análise das duas amostras não foram encontrados outliers cuja exclusão pudesse ter impacto significativo nos resultados. De salientar, ainda, que 12 sujeitos do grupo DS não responderam aos itens do EMBU relativos às memórias de infância do pai, por não terem convivido com ele ou com qualquer outra figura substituta.

Considerando agora as comparações entre grupos ${ }^{10}$, verifica-se que estes diferem de forma estatisticamente significativa em todas as dimensões da EVA (Quadro 2). $O$ grupo DS apresenta valores mais altos na ansiedade $\left[F_{(1,128)}=8.4804, p=.004\right]$ e valores mais baixos nas dimensões confiança nos outros $\left[F_{(1,128)}=9.596, p=.002\right]$ e conforto com a proximidade $\left[F_{(1,128)}=6.336, p=.013\right]$.

10 Foram realizadas ANOVAs One way e teste do Qui-Quadrado para variáveis categoriais e MANOVA para avaliar o efeito do grupo no conjunto das dimensões da vinculação em estudo. Consideraram-se as dimensões conforto com a proximidade, ansiedade e confiança como VD. 
Quadro 2. Comparação entre grupos das pontuações obtidas nas dimensões EVA

\begin{tabular}{lcccccccc}
\hline \multicolumn{7}{c}{ Grupo DS $(\mathrm{n}=65)$} & \multicolumn{2}{c}{ Grupo C $(\mathrm{n}=65)$} \\
\hline & $\mathrm{M}$ & $\mathrm{DP}$ & $\mathrm{M}$ & $\mathrm{DP}$ & $\mathrm{F}$ & $\mathrm{P}$ & $\eta^{2}$ & Obs. Power \\
\hline Ansiedade & 2.757 & .767 & 2,362 & 0.783 & 8.404 & .004 & .062 & .820 \\
Conforto & 3.214 & .476 & 3.492 & 0.548 & 9.596 & .002 & .070 & .867 \\
Confiança & 2.987 & .590 & 3.221 & 0.465 & 6.336 & .013 & .047 & .705 \\
\hline
\end{tabular}

A proporção da variância que se pode atribuir ao efeito do grupo (C ou DS) é, para a dimensão ansiedade, $6.3 \%\left(\eta^{2}=.062\right)$ sendo este valor considerado médio; para dimensão conforto com a proximidade $7 \%\left(\eta^{2}=.07\right)$, sendo este valor também médio; para dimensão confiança $4.7 \%\left(\eta^{2}=.047\right)$, sendo este valor considerado pequeno. A variável grupo está, assim, associada de forma média à variância nas dimensões ansiedade e conforto com a proximidade, enquanto que no caso da dimensão confiança nos outros a associação é fraca.

No que diz respeito ao poder estatístico do estudo, nas dimensões ansiedade e conforto com a proximidade, o valor obtido é superior a .80, referido na literatura como o valor standard para a adequabilidade do modelo ao estudo (Kitler, Menard \& Philips, 2007), enquanto na dimensão confiança nos outros fica abaixo (.705). Ainda assim, embora o poder observado na dimensão ansiedade seja mais baixo do que o valor observado na dimensão conforto, é superior a .80 (Quadro 2).

Considerando as várias dimensões da vinculação em conjunto, as diferenças entre grupos são também estatisticamente significativas $\left[F_{(1,128)}=5.706, p=.001\right]$, o que significa que existe uma diferença entre os dois grupos na medida geral da vinculação, quando se considera a contribuição de cada uma das dimensões em estudo. Estas diferenças evidenciam que os padrões de vinculação se distribuem de forma diferente nos dois grupos. A associação entre a variável grupo e o conjunto das dimensões da vinculação é média $\left(\eta^{2}=.120\right)$, sendo que $12 \%$ da variância no conjunto das dimensões da vinculação se pode atribuir ao efeito do grupo. O poder observado é grande (.943).

A partir dos resultados nas dimensões da EVA, foi possível classificar os sujeitos nas categorias correspondentes aos padrões de vinculação definidos por Bartholomew e Horowitz (1991). Analisando os padrões assim obtidos, verifica-se que as diferenças entre os dois grupos são estatisticamente significativas $\left[\chi^{2}\right.$ $(\mathrm{N}=130)=16.874, p=.001]$. No grupo DS há consideravelmente menos sujeitos classificados como seguros; a penas $38.5 \%(n=25)$ apresentam este padrão, enquanto no grupo $C$ a percentagem é $70.8 \%(n=46)$. Considerando os padrões não seguros, a diferença mais marcada encontra-se ao nível da categoria amedrontado. No grupo DS, 20.0\% ( $n=13$ ) dos sujeitos são assim classificados, enquanto no grupo $C$ encontramos apenas $3.2 \%(n=2)$ dos sujeitos. Tendo em conta as diferentes dimensões do EMBU (memórias de infância), os sujeitos do grupo DS não diferem 
de forma significativa dos sujeitos do grupo C na dimensão suporte emocional do pai. Têm, no entanto, memórias do pai mais relacionadas com a rejeição $\left[F_{(1,}\right.$ $\left.{ }_{116}=22.01 ; p=.000\right]$. Assim, a associação entre a variável grupo e rejeição do pai é grande ( $\left.\eta^{2=} .157\right)$, sendo $15.7 \%$ das diferenças observadas atribuíveis ao efeito da variável grupo. O poder estatístico é também grande (.996). O grupo DS apresenta, também, valores significativamente maiores para o factor sobreprotecção do pai $\left[F_{(1,116)}=12.065 ; p=.001\right]$. A proporção da variância nesta variável explicada pelo factor grupo é de $9.3 \%$ ( $\left.\eta^{2}=.093\right)$, o que corresponde a uma associação moderada entre as variáveis (Quadro 3).

Relativamente às dimensões do EMBU ligadas à mãe, os sujeitos do grupo DS e do grupo C não diferem ao nível do suporte emocional da mãe, mas os primeiros obtêm valores mais altos na rejeição da mãe $\left[\mathrm{F}_{(1,128)}=8.904 ; p=.003\right]$ e na sobreprotecção da mãe $\left[F_{(1,128)}=4.082, p=.045\right)$, de forma semelhante ao que se verificou nas dimensões relativas ao pai (Quadro 3). A associação entre a variável grupo e a rejeição da mãe é moderada $\left(\eta^{2}=.066\right)$, sendo $6.6 \%$ das diferenças encontradas atribuíveis à variável grupo. A associação entre a sobreprotecção da mãe e o factor grupo é pequena $\left(\eta^{2}=.045\right)$, sendo apenas $4.5 \%$ da variância entre os grupos explicada pela inclusão no grupo C ou DS. O poder estatístico observado é adequado no caso da rejeição da mãe, sendo bastante inferior aos .80 considerados como critério standard no caso da sobreprotecção da mãe.

Partindo da estrutura factorial da versão portuguesa do DSQ-4O (Amaral, 2008), verifica-se que os grupos diferem apenas no factor defesas neuróticas $1\left[F_{(1,128)}=5.137\right.$, $p=.025]$. O valor do $\eta^{2}$ é pequeno, sendo a proporção de variância observada neste factor que se atribui ao efeito do grupo (C/DS) apenas de 3.9\%. Relativamente ao poder estatístico, o valor fica também aquém dos .80 convencionados na literatura (Kitler et al., 2007). Este factor é composto pelas defesas supressão, negação e dissociação. A defesa supressão estava incluída no factor composto pelas defesas maturas, na versão original (Andrews, Singh, \& Bond, 1993).

Quadro 3. Comparação entre grupos nas dimensões do EMBU

\begin{tabular}{|c|c|c|c|c|c|c|c|c|}
\hline & \multicolumn{3}{|c|}{$\begin{array}{c}\text { Grupo DS } \\
(\text { Pai } n=53 ; \text { Mãe } n=65)\end{array}$} & \multirow{2}{*}{$\begin{array}{c}\text { Grupo } \\
\text { C } \\
(n=65) \\
\text { DP }\end{array}$} & \multirow[b]{2}{*}{$\mathrm{F}$} & \multirow[b]{2}{*}{$p$} & \multirow[b]{2}{*}{$\eta^{2}$} & \multirow[b]{2}{*}{ Obs. Power } \\
\hline & $M$ & DP & $M$ & & & & & \\
\hline R Pai & 15.287 & 5.430 & 11.406 & 3.561 & 22.031 & .000 & .157 & .996 \\
\hline SE Pai & 14.870 & 4.952 & 15.861 & 4.065 & 1.450 & .231 & .012 & .223 \\
\hline SB Pai & 15.596 & 3.419 & 13.461 & 3.419 & 12.065 & .001 & .093 & .931 \\
\hline R Mãe & 16.000 & 5.491 & 13.419 & 4.241 & 8.904 & .003 & .066 & .842 \\
\hline SE Mãe & 17.120 & 4.746 & 18.523 & 4.552 & 2.934 & .089 & .023 & .398 \\
\hline SB Mãe & 16.000 & 3.943 & 14.297 & 3.943 & 4.082 & .045 & .031 & .518 \\
\hline
\end{tabular}

Legenda: R Pai - rejeição emocional do pai; SE Pai - suporte emocional do pai; SB Pai, sobreprotecção do pai; R Mãe rejeição da mãe; SE mãe - suporte emocional da mãe, SB Mãe - sobreprotecção da mãe 
Considerando individualmente as 20 defesas medidas pelo DSO-40, verifica-se que os grupos apenas diferem na supressão $\left[F_{(1,128)}=7.891 ; p=.006\right]$ e na clivagem $\left[F_{(1,128)}=5.757, p=.018\right]$, defesas onde os sujeitos do grupo DS obtêm pontuações mais altas. A associação entre a variável grupo e as defesas consideradas é pequena no caso da clivagem $\left(\eta^{2}=.043\right)$. A associação entre o grupo e a variável supressão é também pequena ( $\eta^{2}=.058$ ). No caso da clivagem, $4.3 \%$ da variância observada pode ser explicada pelo efeito grupo; no caso da supressão a variabilidade atribuível ao grupo é $5.8 \%$. O poder estatístico observado é baixo no caso da clivagem (.379) e aproxima-se do critério .80 no caso da supressão (.796), definido como standard para a adequabilidade do estudo.

\subsection{Estudo da relação entre as variáveis no grupo DS}

O estudo da relação entre as variáveis no grupo DS $(n=65)$ foi feito, num primeiro momento, com as correlações de Pearson e, num segundo momento, com a técnica de regressão múltipla standard (método enter).

Considerando as correlações entre os factores do EMBU e as dimensões da vinculação medidas pela EVA, a rejeição do pai tem uma correlação positiva estatisticamente significativa com a ansiedade $\left[r_{(53)}=.282, p<.05\right]$, assim como a rejeição da mãe $\left[r_{(65)}=.283, p<.05\right]$. A dimensão confiança nos outros apresenta uma correlação fraca, negativa, com a rejeição da mãe $\left[r_{(53)}=-252, p<.05\right]$. A dimensão da vinculação conforto com a proximidade não se correlaciona com nenhuma das escalas do EMBU.

Tendo em conta a correlação entre as dimensões da EVA e os factores da versão portuguesa do DSQ-40, verifica-se que a ansiedade está correlacionada, de forma estatisticamente significativa e positiva, com todos os níveis defensivos, à excepção das defesas maturas.

A dimensão conforto com a proximidade não se relaciona com nenhum nível defensivo do DSO-4O. A análise dos resultados mostra, ainda, uma correlação negativa entre a confiança nos outros e as defesas imaturas $\left[r_{(64)}=-.363, p<.01\right]$.

Considerando as defesas individualmente, concluímos que a dimensão ansiedade está positiva e moderadamente correlacionada com todas as defesas consideradas como imaturas na versão original da escala. A correlação mais forte é com a clivagem $\left[r_{(64)}=.491, p<.01\right]$ e depois com a projecção $\left[r_{(64)}=.410, p<.05\right]$. Relativamente ao conforto com a proximidade, esta dimensão apenas está correlacionada, de forma estatisticamente significativa, com a sublimação $\left.{ }_{{ }_{(64)}}=.268, p<.05\right]$. Esta é uma defesa matura, de nível mais adaptativo. 
A dimensão confiança nos outros correlaciona-se negativa e moderadamente com o isolamento $\left[r_{(64)}=-.417, p<.01\right]$ e o acting out $\left[r_{(64)}=-437, p<.01\right]$. Esta dimensão apresenta correlações também negativas, estatisticamente significativas, com a clivagem $\left[r_{(64)}=-.329, p<.01\right]$, a negação $\left[r_{(64)}=-.331, p<.01\right]$, a somatização $\left[r_{(64)}\right.$ $=-.277, p<.01]$, a idealização $\left[r_{(64)}=-.318, p<.05\right]$ e a antecipação $\left[r_{(64)}=-250, p<.05\right]$.

Considerando agora o poder preditivo das memórias de infância e dos mecanismos de defesa nas dimensões da vinculação, foram tomados como preditores as variáveis rejeição da mãe e clivagem, uma vez que, analisando as correlações obtidas, se verifica que a rejeição da mãe (EMBU) está correlacionada com a ansiedade e confiança nos outros (EVA), sugerindo que este é o factor do EMBU que mais contribui para as pontuações nas diferentes dimensões da vinculação. Do ponto de vista teórico, a rejeição da mãe é, também, considerada um dos principais factores na origem da insegurança na vinculação. Também a clivagem é a defesa que apresenta uma correlação mais elevada com a ansiedade, estando também correlacionada com a confiança. A clivagem é, também do ponto de vista clínico, o mecanismo de defesa principal associado à organização Borderline (Bergeret, Bécache, Boulanger, \& Chartier, 1998; Braconnier et al., 2007; Kernberg, 1977) e à problemática da DS (e.g. Bergeret et al, 1998; Braconnier et al, 2007). Neste sentido, foram incluídos no modelo de regressão linear múltipla a rejeição da mãe e a clivagem, procurando medir-se o impacto destas variáveis nas dimensões de vinculação. Considerando as duas variáveis em simultâneo, procurou-se ter uma visão mais clara da sua contribuição conjunta e, analisando o impacto relativo de cada uma das variáveis, verificar qual o melhor estimador das diferenças individuais observadas a nível das dimensões da vinculação. A dimensão conforto com a proximidade não se correlaciona com nenhum factor do EMBU, não sendo por isso aqui analisada.

Os pressupostos estatísticos para a aplicação da regressão (Tabachnick \& Fidell, 1996; Moroco, 2003) foram verificados, nomeadamente a normalidade, homocedasticidade dos resíduos e a multicolinearidade entre as VIs (VIF=1.065).

Relativamente à dimensão ansiedade, o modelo apresentado explica $28.5 \%$ da variância, sendo considerado estatisticamente significativo $\left(F_{(1,63)}=12.164, p=.000\right)$.

Numa análise aos coeficientes de regressão, verifica-se que apenas a clivagem tem um poder preditivo estatisticamente significativo $\left(\beta=.437 ; \mathrm{t}_{(62)}=3.908, p<.000\right)$ na dimensão ansiedade da vinculação, sendo, portanto, um melhor estimador das diferenças observadas nessa dimensão. Considerando a dimensão confiança nos outros, o modelo apresentado explica $16.6 \%$ da variância observada nessa dimensão, sendo estatisticamente significativo $\left.\left[F_{(1,62)}=6.062\right), p=004\right]$.

Analisando os coeficientes de regressão verifica-se que tanto a rejeição da mãe $\left[\beta=-.267, \mathrm{t}_{(62)}=-2.055, p=.031\right]$ como a clivagem $\left[\beta=-.248, \mathrm{t}_{(62)}=-2.14, p=-.048\right]$ são 
preditores da confiança nos outros. Há que realçar que o sentido da relação entre as variáveis é negativo, ou seja, o aumento da rejeição da mãe e da clivagem determinam uma menor confiança nos outros.

\section{Discussão dos resultados}

O controlo dos níveis de psicopatologia, medidos pelo BSI, evidencia que em ambos os grupos os valores médios obtidos se aproximam dos valores normativos (Canavarro, 2006), não apresentando sintomatologia psicopatológica grave. O grupo DS, inclusivamente, obteve resultados inferiores aos valores médios encontrados por Canavarro (1999) para a população com perturbação emocional. Este dado pode ser compreendido considerando o facto destes sujeitos se encontrarem em tratamento. Comparando os dois grupos, contudo, verifica-se que os sujeitos DS apresentam mais sintomas de depressão, obsessão-compulsão e psicoticismo. Apresentam também resultados mais elevados no Índice Geral de Sintomas (IGS) e Índice de Sintomas Positivos (IGS). De salientar, no entanto, que os valores mais elevados observados na escala de psicoticismo e depressão no grupo DS podem estar relacionados com o uso excessivo da clivagem, tal como tem sido descrito nos fenómenos borderline (Bergeret et al., 1998; Braconnier et al., 2007; Kernberg, 1977).

Os resultados da comparação entre o grupo C e o grupo DS nas dimensões da vinculação (ansiedade, conforto com a proximidade e confiança nos outros), estão na mesma linha dos obtidos por Thorberg e Lyvers (2006): os sujeitos DS obtêm pontuações médias mais elevadas na dimensão ansiedade e pontuações mais baixas em confiança e conforto. Estes resultados sugerem que estes sujeitos vivem de forma mais intensa o medo de abandono, ou de não ser amados, pontuando por isso mais alto na ansiedade. Sentem-se, ainda, pouco confortáveis com a proximidade e a intimidade e não confiam na disponibilidade dos outros em situações de necessidade. Quando se comparam os grupos considerando como variável dependente o conjunto das três dimensões da vinculação da EVA, que estão subjacentes a diferentes padrões de vinculação, a diferença entre os grupos é significativa, sendo que a variável grupo explica $12 \%$ da variância observada no conjunto das três dimensões. Este resultado é particularmente interessante, uma vez que mostra que as diferenças entre grupos resultam de um determinado "arranjo" específico das diferentes dimensões da vinculação que subjazem a determinado padrão, e não apenas a diferenças que se verificam em cada uma dessas dimensões quando consideradas individualmente.

Transformando estas pontuações nas quatro categorias de Bartholomew e Horowitz, (1991), verificamos que, de facto, no grupo DS a percentagem de indivíduos 
classificados como seguros é significativamente mais baixa do que no grupo C. A maioria dos sujeitos é classificada como não segura. A combinação de modelos internos dinâmicos negativos de si e do outro, corresponde, na categorização de Bartholomew e Horowitz (1991), ao padrão de vinculação amedrontado. É nesta categoria que a diferença de proporção entre os dois grupos é mais evidente $(n=13$ no grupo DS e $n=2$ no grupo $C$ ). Os resultados indicam que estes indivíduos utilizam estratégias de desactivação, tipicamente evitantes, mas sem sucesso (Schindler et al., 2005). Continuam a perceber as ameaças como os indivíduos ansiosos (hiperactivação do sistema de vinculação), mas não concebem a aproximação à figura de vinculação como uma estratégia para lidar com o sofrimento. Por hipótese, na ausência deste tipo de estratégias eficazes, o consumo de substâncias pode ser visto como uma forma artificial de desactivar o sistema de vinculação, sendo uma tentativa de lidar com a elevada ansiedade sem recorrer ao outro, utilizando um objecto controlável, que cumpre a função de base segura (Ainsworth, 1967), na regulação afectiva. Do ponto de vista da teoria da vinculação, estes dados suportam a hipótese da DS poder ser vista como uma forma de lidar com a ansiedade da vinculação, diminuir o sofrimento emocional e regular as relações interpessoais (e.g. Schindler et al., 2005).

O padrão desligado do modelo de Bartholomew e Horowitz (1991) pode estar subrepresentado em relação à sua prevalência na população DS. Efectivamente, e uma vez que os indivíduos classificados como desligados têm modelos positivos de si e modelos negativos dos outros, será de prever que se envolvam menos em tratamento do que os sujeitos com outros padrões de vinculação. Caspers et al. (2006) mostraram que os sujeitos classificados como evitantes na AAl eram os que menos se envolviam em tratamento, embora apresentassem problemas significativos de abuso de substâncias. Apesar da categoria evitante da AAl e desligada do modelo de Bartholomew e Horowitz (1991) não terem correspondência directa, a falta de confiança necessária para procurar apoio junto de uma figura de vinculação, em tempos de stress e sofrimento, é característica de ambas as categorias. De qualquer modo, e uma vez que todos os sujeitos da amostra DS estão a ser seguidos por instituições ligadas ao tratamento da DS, é possivel a existência de um enviesamento dos dados, no sentido da sub-representação dos individuos DS na categoria desligado.

Relativamente às escalas do EMBU, os sujeitos do grupo DS reportam mais rejeição de ambos os pais, mas não se distinguem dos sujeitos do grupo $\mathrm{C}$ em termos de suporte emocional. A pontuação obtida pelos sujeitos do grupo $\mathrm{C}$ fica abaixo da pontuação da média observada no estudo de Canavarro (1996), o que pode estar relacionado com o facto do grupo $\mathrm{C}$ ser de um estatuto socioeconómico muito mais baixo do que o desse estudo. Além de diferir a nível da rejeição de ambos os pais, o grupo DS apresenta também níveis mais elevados de sobreprotecção, 
o que se enquadra no estilo de cuidados parentais ambivalentes, marcados pela rejeição e falta de afecto e apoio mas, também, pela sobreprotecção e controlo excessivo sem afecto (affectionless control), referidos na literatura (e.g. Andersson \& Eiseman, 2003; De Jong et al., 1991; Emmelkamp \& Heeres, 1988; Shweitzer \& Lawton, 1989). Assim, e uma vez que a maioria dos sujeitos em estudo é classificado em padrões não seguros de vinculação, os resultados estão de acordo com a concepção de que representações ambivalentes, frias e rejeitantes dos pais se associam a padrões de vinculação não seguros (e.g. Canavarro et al., 2006; Mickelson et al., 1997).

No grupo DS, 12 dos sujeitos não responderam às questões sobre o pai, porque não conviveram com ele ou com qualquer outra figura substituta. No grupo C nenhum dos sujeitos abordados se encontrava nesta situação. Efectivamente, este tipo de ambiente familiar marcado pela ausência, perda e patologias do luto têm sido frequentemente associadas à DS (e.g. Braconnier et al., 2007).

A dimensão ansiedade da vinculação do adulto está correlacionada com a rejeição do pai e da mãe. As restantes práticas de cuidados parentais (suporte emocional e sobreprotecção) não apresentam correlações significativas com esta dimensão. Estando a alta ansiedade relacionada com modelos internos dinâmicos negativos do self, que se formam a partir da interacção precoce com o cuidador, os resultados corroboram a hipótese de que estes modelos se formam na interacção do dia-a-dia com os cuidadores, e são actualizados nas relações estabelecidas na idade adulta (Bowlby, 1958; Canavarro et al., 2006; Fonagy, 2001).

A dimensão confiança nos outros está negativamente correlacionada com a rejeição da mãe. A percepção, por parte da criança, de uma mãe pouco responsiva e sensitiva, levaria a que esta construísse modelos internos dinâmicos negativos dos outros, não tendo confiança na sua disponibilidade e capacidade para estar presente em tempos de necessidade. A rejeição do pai não se correlaciona com a confiança nos outros. Esta diferença entre o pai e a mãe pode ser entendida pela diferença de papéis, uma vez que a mãe é mais precocemente a figura a quem a criança mais recorre em tempos de necessidade, sendo também aquela que mais investe na criação de um laço com a criança (Ainsworth, 1967); muitas vezes, o contacto com o pai é mais escasso e assenta noutros aspectos que não remetem para a sensitividade às necessidades da criança e capacidade para Ihes responder, ainda que a natureza desta vinculação permaneça pouco clara.

O conforto com a proximidade não está correlacionado com nenhuma dimensão do EMBU. O mais previsivel seria que o conforto com a proximidade se associasse ao suporte emocional dos pais. Os sujeitos do grupo DS obtêm pontuações baixas quer para o suporte emocional de ambos os pais, quer para o conforto com a proximidade. Assim, os dados sugerem que a memória do suporte emocional na 
infância não é muito frequente, não estando, no caso do grupo DS, pela sua pouca relevância no estilo de cuidados parentais, na origem da formação de modelos internos dinâmicos positivos de si ou do outro.

No que concerne às defesas, o único factor em que os sujeitos do grupo DS pontuam significativamente acima dos sujeitos do grupo C é o factor defesas neuróticas, composto pela supressão, negação e dissociação. Este factor, na versão portuguesa (Amaral, 2008), contém duas defesas imaturas da estrutura factorial da versão original (Andrews et al., 1993), a negação e dissociação, e uma defesa considerada matura, a supressão. No caso da amostra em estudo, a maioria dos sujeitos foi classificada em categorias que têm subjacentes modelos internos dinâmicos negativos do outro (desligado e amedrontado), a que se associa uma desvalorização das ameaças e do próprio sofrimento, o que parece estar, também, subjacente à utilização dos mecanismos de defesa que compõem este factor de defesas neuróticas.

No entanto, os resultados não evidenciam uma maior utilização de defesas imaturas no grupo DS, o que parece contrastar com os dados obtidos no estudo de Comings et al. (1995). Por hipótese, este resultado pode estar relacionado com o facto de todos estes sujeitos DS se encontrarem em tratamento, o que poderá implicar um funcionamento mais saudável.

Considerando os mecanismos de defesa específicos, os sujeitos do grupo DS pontuam significativamente mais alto na clivagem/polarização e na supressão. A supressão faz parte do factor defesas neuróticas e pode ser associada a estratégias secundárias de regulação do afecto, segundo a teoria da vinculação, sendo fácil fazê-la corresponder, em termos conceptuais, à exclusão defensiva. A clivagem pode ser, tal como a dissociação, associada à segregação de sistemas principais. Este mecanismo de defesa é de extrema importância na compreensão da DS, sendo considerado o mecanismo central da organização limite da personalidade (Braconnier et al., 2007; Kernberg, 1977), do mesmo modo que estas duas perturbações (DS e estado-limite) apresentam uma elevada comorbilidade (e.g. Conrod \& Stewart, 2005; Trull, Sher, Minks-Brown, Durbin, \& Burr, 2000).

A dimensão ansiedade, por outro lado, está positivamente correlacionada com todos os factores da versão portuguesa do DSQ-40 (Amaral, 2008), à excepção do factor defesas maturas. Isto é, os indivíduos mais ansiosos utilizam preferencialmente estratégias defensivas pouco adaptativas para fazer face à angústia.

O conforto com a proximidade apenas se relaciona com um mecanismo de defesa maturo, a sublimação. Este dado é consistente com o pressuposto de Bowlby (1980) que o conforto com a proximidade, base de um padrão seguro de vinculação, não se relaciona com muitos mecanismos de defesa. Os indivíduos 
seguros (com alta confiança nos outros) tenderão a recorrer à proximidade com a figura de vinculação em situações de stress, sendo esta a sua estratégia primária de regulação afectiva. Por outro lado, a segurança da vinculação (para a qual o conforto com a proximidade contribui fortemente, juntamente com a confiança) está associada ao desenvolvimento da capacidade de mentalização e simbolização, bem como à capacidade do sujeito se desligar da necessidade concreta da presença do outro, passando a ser mais autónomo e capaz de se auto-regular afectivamente (Fonagy, 2001). Uma maior energia fica disponível para investir a outros níveis, de um modo geral. Assim, a capacidade de sublimação surge ligada ao desenvolvimento de aspectos mais saudáveis do ego, no contexto de uma relação de vinculação marcada pela segurança.

A confiança nos outros está correlacionada negativamente com as defesas imaturas da versão portuguesa do DSQ-40 no seu conjunto e com várias defesas específicas. A incapacidade de recorrer ao outro e senti-lo como disponível em tempos de necessidade surge, assim, associada a estratégias secundárias de regulação do afecto, mais regressivas, tal como sucede com a ansiedade.

De um modo geral, pode dizer-se que o conforto com a proximidade está associado a um funcionamento mental mais saudável e adaptativo, enquanto a baixa confiança no outro (modelos internos dinâmicos negativos do outro) e a alta ansiedade (modelo interno dinâmico negativo de si) se relacionam com um funcionamento menos adaptativo, com recurso a defesas mentais mais regressivas e dispendiosas para o ego.

Na análise da regressão, quanto à dimensão ansiedade, verificou-se que apenas a clivagem tem um poder preditivo. A rejeição da mãe fica no limiar de significância estatística. Uma vez que os mecanismos de defesa emergem na primeira infância (Freud, 1923/1969), pode especular-se que a utilização de determinados mecanismos de defesa se constitua como um factor que contribui para a continuidade entre as experiências na infância e a vinculação do adulto. A clivagem remete para instabilidade na representação do eu e dos outros, oscilando entre o negativo e o positivo, dando algum sentido à importância da dimensão "psicoticismo" encontrada nestes sujeitos (BSI). De qualquer modo, este funcionamento pode estar relacionado com a existência de modelos internos dinâmicos opostos relativos a si e aos outros, desenvolvidos em momentos diferentes, que são activados alternadamente (segregação de sistemas principais, segundo Bowlby, 1980). Por exemplo, um modelo interno dinâmico negativo formado na infância, pode ficar sempre latente, enquanto outros modelos internos dinâmicos, mais positivos, são desenvolvidos posteriormente, noutros contextos (Bowlby, 1980). Ainda que os modelos internos dinâmicos negativos, formados na infância, estejam "adormecidos", isto é, não conscientes, o sujeito que vive este paradoxo interno será 
sempre marcado pela instabilidade e dificuldades de regulação afectiva. Assim, a existência destes modelos internos dinâmicos discrepantes está na origem, segundo o modelo proposto, de dúvidas em relação ao próprio valor, receio de ser abandonado e percepção aumentada de ameaças à relação; em suma, da ansiedade na vinculação. Estes resultados vão no mesmo sentido dos obtidos por Lopez (2001) que verificou que altos niveis de ansiedade estavam associados à clivagem da imagem do outro e do self. Relativamente à dimensão confiança, verificou-se que tanto a rejeição da mãe como a clivagem determinam menores pontuações nesta dimensão; experiências com uma mãe rejeitante associam-se a representações do outro como pouco fiável e incapaz de dar conforto em tempos de necessidade. A clivagem determina a dimensão confiança nos outros de modo semelhante ao que foi descrito para a dimensão ansiedade: a instabilidade relativa à imagem do outro está na origem da incapacidade de construir uma representação estável desse outro, como alguém capaz de responder às necessidades e dar conforto em situações de sofrimento. A clivagem pode, assim, ser compreendida como um mecanismo de defesa associado a dimensões que determinam padrões não seguros de vinculação.

\section{Conclusões}

De sublinhar, em primeiro lugar, a existência de diferenças estatisticamente significativas nas três dimensões da vinculação aqui medidas: ansiedade, conforto com a proximidade e confiança nos outros. Assim, e de um modo geral, o grupo DS é caracterizado por dimensões da vinculação subjacentes a padrões não seguros, particularmente o padrão amedrontado de Bartholomew e Horowitz (1991), o que se relaciona preferencialmente, de acordo com o construto (Schindler et al., 2009), com as memórias de experiências precoces de rejeição e a utilização de estratégias secundárias de regulação do afecto menos adaptativas (por oposição ao recurso à figura de vinculação).

As diferenças entre grupos nas memórias de infância corroboram os resultados já encontrados noutros estudos, sendo as memórias dos sujeitos DS mais marcadas pela sobreprotecção e rejeição (Andersson \& Eismann, 2003; De Jong et al., 1991; Emmelkamp \& Heeres, 1988; Shweitzer \& Lawton, 1989; Zimmer-Hofler \& Kooyman, 1996). As diferenças entre grupos, no que toca ao suporte emocional, não são significativas; os sujeitos do grupo C, de estatuto socioeconómico baixo, têm pontuações mais baixas do que a amostra estudada por Canavarro (1996). O grupo DS obtém pontuações a nível dos mecanismos de defesa que diferem dos resultados verificados noutros estudos, com as defesas maturas a obterem pontuações superiores (Commings et al., 1995; Raketic et al., 2009). Ainda assim, 
quando se consideram as defesas específicas, os sujeitos DS recorrem mais à supressão e à clivagem. A pontuação elevada na clivagem relativamente ao grupo de controlo é particularmente interessante, uma vez que este mecanismo de defesa está, teoricamente, relacionado com o funcionamento mental característico da DS, considerado pelos autores de orientação analítica (Bergeret et al., 1998; Braconnier et al., 2007), como um quadro marcado pela organização Borderline da personalidade, com aspectos psicóticos, depressivos, lutos patológicos e instabilidade psíquica (Bergeret et al., 1998; Braconnier et al., 2007; Henriques \& Paixão, 2009; Kernberg, 1977).

A análise da regressão permitiu ter uma visão conjunta das memórias de experiências precoces (mais concretamente da rejeição da mãe) e da utilização da clivagem enquanto preditor das dimensões da vinculação do adulto. Ambas as variáveis contribuem parcialmente para estas dimensões; a clivagem parece ser um melhor estimador de maior ansiedade da vinculação no adulto e menor confiança nos outros. Estes resultados sugerem que os mecanismos de defesa poderão estar na base da continuidade entre a experiência precoce e a vinculação do adulto, o que poderá ser útil na compreensão do funcionamento mental característico dos sujeitos com DS.

\section{Referências bibliográficas}

Ainsworth, M. (1967). Infancy in Uganda: Infant care and the growth of love. Baltimore: Johns Hoppkins University Press.

Amaral, I. (2008). Versão Portuguesa do DSO-40.Tese de Mestrado. Faculdade de Psicologia e Ciências da Educação. Coimbra: Universidade de Coimbra.

American Psychiatric Association (2002). Manual de Diagnóstico e Estatistica das Perturbações Mentais - texto revisto. ( $4^{a}$ ed). Lisboa: Climepsi.

Andersson, P., \& Eismann (2003). Parental rearing and individual vulnerability to drug addiction: a controlled study in a swedish sample. Nord Journal of Psychiatry, 57,147-156.

Andrews, G., Singh, M., \& Bond, M. (1993). The defence style questionnaire. The Journal of nervous and mental diseases, 181 (4), 246-256.

Arrindell, W.A., Emmelkamp, P.M.G., Monsma, A., \& Brilman, E (1983). Psychiatric evaluation of an inventory for assessment of parental rearing practices. Acta Psychiatrica Scandinavia, 67, 163-177.

Barone, L., Lingiardi V., \& Maffei, C. (1996) Attachment patterns and defense mechanism in personality disorders: a preliminar study. European Psychiatry, 11, 238-248.

Bartholomew, K., \& Horowitz, L.M. (1991). Attachment styles among young adults: A test of a four-category model. Journal of Personality and Social Psychology, 61, 226-244.

Bergeret, J., Bécache, A., Boulanger., J-J., Chartier, P. (1998). Psicologia Patológica: Teórica e clínica. Lisboa: Climepsi Editores. 
Bernier, A. \& Dozier, M. (2002). The client-counselor match and the corrective emotional experience: Evidence from interpersonal and attachment research. Psychotherapy: Theory/ Research/ Practice/ Training, 39, 32-43.

Bernstein, D.P., Stein, J.A., \& Handelsman, L. (1998). Predicting personality pathology among adult patients with substance use disorders: effects of childhood maltreatment. Addictive Behaviors, 6, 855-868.

Bond, M., Gardner ST., Christian J., \& Sigal J.J. (1983). Empirical study of self-rated defense styles. Arch Gen Psychiatry, 40, 333-338.

Bowlby, J. (1958). The nature of the child's tie to his mother. International Journal of Psychoanalysis, 39, 350-373.

Bowlby, J. (1980). Attachment and loss. New York: Basic Books.

Bowlby, J. (1988). A secure base: Parent-child attachment and healthy human development. New York: Basic Books.

Braconnier, A., Corbobesse, F., Deschamps, F., Duriez, N., Gailard-Janin, N., Jourbet-Chain, C., Laugier, F., \& Saint-Georges, C. (2007). Manual de Psicopatologia. Lisboa: Climepsi Editores.

Branstetter, S.A., Bower. E.H., Kamien, J., \& Amass, L.(2008). A history of sexual, emotional, or physical abuse predicts adjustment during opióide maintenance treatment. Journal of Substance Abuse Treatment, 34, 208-214.

Bretherton, I. (1985). Attachment theory: Retrospect and prospect. In I. Bretherton \& E. Waters. Growing points of attachment theory and research. Monograph of the Society for Research in Child Development, 209, 3-35.

Canavarro, M.C. (1999). Inventário de Sintomas Psicopatológicos - B.S.I. In M. Simões, M. Gonçalves, \& L. Almeida (Eds.). Testes e Provas Psicológicas em Portugal (Vol. II) (pp. 96-109), Braga: SHO.

Canavarro, M.C. (1996). A avaliação das práticas educativas através do EMBU: estudos psicométricos. Psychologica, 16, 5-18.

Canavarro, M.C., Dias, P., \& Lima., M. (2006). A avaliação da vinculação do adulto: uma revisão crítica a propósito da aplicação da adult attachment scale ASS-R na população portuguesa. Psicologia, XX (1), 11-36.

Caspers, K.M., Yucuis, R., Troutman, B., \& Spinks, R. (2006). Attachment as an organizer of behavior: implications for substance abuse problems and willingness to seek treatment. Substance abuse treatment, Prevention, and policy, 1, 1-10.

Collins, N., \& Read, S. (1990). Adult attachment relationships, working models and relationship quality in dating couples. Journal of Personality and Social Psychology, 58, 644-683.

Collins, N. (1996). Working Models of attachment: Implications for explanation, emotion, and behavior, Journal of Personality and Social Psychology, 74, 810-832.

Comings, D.E., McMurray, J., Johnson, P., Dietz, \& Muhleman, D. (1995). Dopamine D2 Receptor Gene (DRD2) Haplotypes and the defense style questionnaire in substance abuse, Tourette syndrome and controls. Biological Psychiatry, 37, 798-805.

Conrod, P.J., \& Stewart, S.H. (2005). A critical look at dual focused Cognitive-Behavioral treatments for comorbid substance use and psychiatric Disorders: Strengths, Limitations and Future Directions. Journal of cognitive psychotherapy, 16, 261-284.

Cooper, M.L., Shaver, P.R., \& Collins (1998). Attachment styles, emotion regulation and adjustment in adolescence. Journal of Personality and social Psychology, 74, 1380-1397. 
De Jong, C.A., Harvefeld, F.M., van der Wlelen, G.E.M., \& van der Stank, C.P F. (1991). Memories of parental rearing in alcohol and drug addicts: A comparator study. International Journal of Addiction, 23, 207-216.

Derogatis, L. R., \& Spencer, P. M. (1982). The Brief Symptom Inventory (BSI): Administration, and Procedures Manual-I. Baltimore, MD: Clinical Psychometric Research.

Emmelkamp, P.M.G. \& Heeres, H. (1988). Drug addiction and parental rearing style: A controlled study. International. Journal of Addiction. 23(2), 207-216.

Finzi-Dottan, R., Cohen, O., Iwaniec, D., Sapir, Y., \& Weitzman, A. (2003). The drug user husband and his wife: Attachment styles, family cohesion and adaptability. Substance Use and Misuse, 38, 271-292.

Fonagy, P. (2001). Attachment theory and psychoanalysis. New York: Other Press.

Fonagy, P., Leigh, T., Steele., H., Kennedy, R., \& Matoon, G. (1996). The relation of attachment status, psychiatric classification and response to psychotherapy. Journal of counselling and consulting psychology, 64, 22-31.

Freud, S. (1923/1969). O Ego e o Id. In P. Maudreira (Ed.), Edição Satandard Brasileira das Obras Completas de Sigmund Freud. Vol. XIX (pp. 13-83). Rio de Janeiro: Imago Editora.

Hazan, C., \& Shaver, P.R. (1987). Romantic love conceptualized as an attachment process. Journal of Personality and Social Psychology, 52, 511-524.

Henriques, A., \& Paixão, R. (2009). Padrões de Consumo de Álcool em Programas de Substituição de Opiáceos, Toxicodependências, 15 (3), 39-49.

Insel, T.R. (2003). Is social attachment an addictive disorder? Physiology and Behavior, 79, 351-357.

Kernberg, O.F. (1977). The structural diagnosis of Borderline personality organization. In P. Hartocollis (Ed.), Borderline Personality disorders: The concept, the syndrome, the patient (pp.87-121). New York: International University Press.

Kittler, J.E., Menard, W., \& Phillips, K.A. (2007). Weight concerns in individuals with body dimorphic disorder. Eating Behaviors, 8, 115-120.

Levy, K.N., Blatt, S.J., \& Shaver, P.R. (1998). Attachment styles and parental representation. Journal of Personality and Social Psychology, 74, 407-419.

Lopez, G. (2001). Adult Attachment Orientations, Self-Other Boundary Regulation, and splitting tendencies in a college sample. Journal of Counseling Psychology, 48, 440-446.

Main, M., \& Hesse, E. (1990). Parent's unresolved traumatic experiences are related to infant disorganized attachment status: Is frightened and/or frightening parental behavior the linking mechanism? In M. Greene, D. Chiceti, \& E.M. Cummings (Eds.), Attachment and Preschool years: Theory, Research, and Intervention (pp.161-182). Chicago: University of Chicago Press.

Main, M., Kaplan, N., \& Cassidy, J. (1985). Security in infancy, childhood, and adulthood: a move to the level of representation. In I. Bretherton \& E. Waters (Eds.). Growing points of attachment theory and research in Child Development (pp. 66-104). Chicago: University of Chicago Press.

McNally, A. M., Palfai, T. P., Levine, R. V., \& Moore, B. M. (2003). Attachment dimensions and drinking-related problems among young adults: the meditational role of coping motives. Addictive Behaviors, 28, 1115-1127.

Mickelson, K.D., Kessler, R.C., \& Shaver, P.R. (1997). Adult attachment in a nationally representative sample. Journal of Personality and Social Psychology, 73, 1092-1106.

Mikulincer, M., \& Shaver, P.R. (2007). Attachment in adulthood. New York: Guilford. 
Moroco, J. (2003). Análise estatística com utilização do SPSS. Lisboa: Sílabo.

Parker G, Tupling H, \& Brown L.B. (1979). A Parental Bonding Instrument. Br. J. Med. Psychol., $52,1-10$.

Paulli-Pot, U., \& Mertesacker, B. (2009). Affect expression in mother-infant interaction and subsequent attachment development. Infant Behavior and Development, 37, 1-8.

Perris, C., Jacobson, L., Lindstorm, H., von knorring, L., \& Perris, H. (1980). Development of a new inventory for accessing memories of parental rearing behaviour. Acta Psychiatrica Scandinavica, 61, 265-274.

Raketic, D., Kovacevic, M., \& Djuric (2009). Women addiction and defense mechanism style. European Psychiatry, 24, 35-78.

Riggs, A., Paulson, A., Tunnell, E., Sahl, G., Atkinson, H., \& Ross, C.A. (2007). Attachment, personality, and psychopathology among adult inpatients: self-reported romantic attachment style versus Adult Attachment Interview states of mind. Development and Psychopathology, 19, 263-291.

Riggs, S.A., \& Jacobvitz, D. (2002). Expectant parents' representations of early attachment relationships: associations with mental health and family history. Journal of Consulting and Clinical Psychology, 70, 195-204

Rodrigues, A., Figueiredo, B., Pacheco, A., Costa, R., Cabeleira, C., \& Magarinho, R. (2004). Memórias de cuidados da infância, estilo de vinculação e qualidade da relação com pessoas significativas: Estudo com grávidas adolescentes. Análise Psicológica, $22,643-665$.

Rosenstein, D.S., \& Horowitz, H.A. (1996). Adolescent attachment and psychopathology. Journal of Consulting and Clinical Psychology, 64, 244-253.

Schindler, A., Thomasius, R., Petersen, K., \& Sack, P.M. (2009). Heroin as an attachment substitute? Differences in attachment representations between opióide, ecstasy and cannabis abusers. Attachment and Human Development, 11, 307-330.

Schindler, A., Thomasius, R., Sack, P.M., Gemeinhardt, B., Kustner, U.J., \& Eckert, J. (2005) Attachment and substance use disorders: a review of the literature and a study in drug dependent adolescents. Attachment \& Human Development, 7, 207-228.

Shaver, P.R., \& Milkulincer, M. (2005). Attachment theory and research: Resurrection of the psychodynamic approach to personality. Journal of Research in Personality, 39, 22-45.

Shweitzer, R.D., \& Lawton, A.P. (1989). Drugs Abuser's perceptions of their parents. British Journal of addictions, 84, 309-314.

Tabachnik, B.G., \& Fidell, L.S. (1996). Using multivariate statistics.. New York: Harper Collins.

Teixeira, J.M., \& Fernandes, R.P. (2005). Comorbilidade psiquiátrica em heroinodependentes de rua. Saúde Mental, 7, 11-14.

Thorberg, F.A. \& Lyvers, M. (2006). Attachment, fear of intimacy and differentiation of self among clients in substance disorder facilities. Addictive Behaviors, 31, 732-727.

Trull, T. J., Sher, K. J., Minks-Brown, C., Durbin, J., \& Burr, R. (2000). Borderline personality disorder and substance use disorders: A review and integration. Clinical Psychology Review, 20, 235-253.

Vaillant, G. E. (1994). Ego mechanisms of defence and personality psychopathology. Journal of Abnormal Psychology, 103, 44-50.

Zimmer-Hofler, D. \&. Kooyman, M. (1996). Attachment transition and therapeutic BondingAn integrative approach. Journal of substance abuse treatment, 13, 511-519. 


\section{Attachment, Child Memories and Defence Styles in Drug Abusers: a comparative and multivariate study}

The present work reports on a study of attachment dimensions in drug abusers, as well as other related variables as childhood memories about relationship with parents and defence mechanisms. The research was conducted in two studies: one comparing drugs abuser's $(n=65)$ with a match group (in terms of sex, age and education level) of individuals from the common population $(n=65)$ and other analysing the relationship between attachment styles, child memories and specific defence mechanisms in the drug abusers group. In the first study differences between groups were significant in terms of attachment, child memories and specific defences, namely splitting and suppression. In the second study results show that memories of mother rejection and splitting have a significant preditor effect in the dimensions of attachment in drug abusers. In this group splitting defence seems to be a better predictor of these dimensions.

KEY-WORDS: Attachment, Internal Working Models, Substance Dependence, Memories of Parental Rearing Behaviour, Defence Profiles

\section{Attachement, souvenirs d'enfance et style défensif dans la toxicoma- nie: étude comparative et multidimensionnelle}

L'objectif de ce travail est l'étude de l'attachement dans la toxicomanie, considérant la relation de cette problématique avec les souvenirs d'enfance relies aux parents et les styles défensifs des sujets. Dans an premier étude un group des toxicomanes $(n=65)$ est comparé avec un group de sujets de la population commun $(n=65)$ équivalente en termes de sexe, âge et éducation. Les différences entre ces deux groups sont significatif au niveau de l'attachement, des souvenirs d'enfance et des mécanismes défensives spécifiques comme la clivage et la suppression. Dans an second étude, la relation entre ces variables dans le group toxicomane a mis en évidence que la mémoire de la rejection de la mère et le clivage on en effet prédicteur au niveau des dimensions de l'attachement. Dans ce cas, le clivage constitue un estimateur consistent de ces dimensions.

MOTS-CLÉS: Attachement, Modèles de travaille interne, Toxicomanie, Souvenirs de soins parentaux, Styles défensives 